\title{
Viral-Assisted Assembly and Photoelectric Response of Individual Au/CdSe Core-Shell Nanowires
}

\section{Citation}

Joo, John H., Juan F. Hodelin, Evelyn L. Hu, and Elaine D. Haberer. 2012. Viral-assisted assembly and photoelectric response of individual $\mathrm{Au} / \mathrm{CdSe}$ core-shell nanowires. Materials Letters 89:347-350.

\section{Published Version}

doi:10.1016/j.matlet.2012.09.001

\section{Permanent link}

http://nrs.harvard.edu/urn-3:HUL.InstRepos:11006843

\section{Terms of Use}

This article was downloaded from Harvard University's DASH repository, and is made available under the terms and conditions applicable to Open Access Policy Articles, as set forth at http:// nrs.harvard.edu/urn-3:HUL.InstRepos:dash.current.terms-of-use\#OAP

\section{Share Your Story}

The Harvard community has made this article openly available.

Please share how this access benefits you. Submit a story.

\section{Accessibility}


Title: Viral-assisted assembly and photoelectric response of individual Au/CdSe core-shell nanowires Authors: John H. Joo ${ }^{\mathrm{a}}$, Juan F. Hodelin ${ }^{\mathrm{b}}$, Evelyn L. Hu ${ }^{\mathrm{a}}$, and Elaine D. Haberer ${ }^{\mathrm{c}}$ Affliations: ${ }^{\text {a S}}$ Shool of Engineering and Applied Sciences, Harvard University, Cambridge, MA 02138

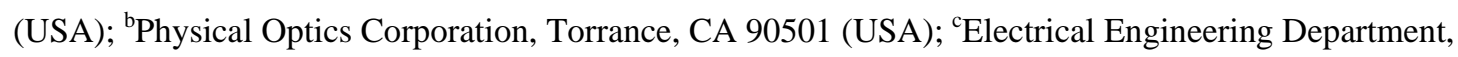
Materials Science and Engineering Program, University of California, Riverside, CA 92521 (USA)

Corresponding Author: Elaine D. Haberer, Electrical Engineering Department, Materials Science and Engineering Program, University of California, Riverside, CA 92521 (USA)

Tel: 951.827.7174; FAX: 951.827.2425; E-mail: haberer@ucr.edu

Abstract: Individual viral-templated Au/CdSe core-shell nanowires were synthesized and electrically characterized at room temperature. The Au nanowire cores were constructed using a genetically-modified filamentous M13 bacteriophage as a scaffold. Au nanoparticles were selectively bound to the viruses and used as seeds for electroless deposition, forming continuous Au nanowires. The nanocrystalline CdSe shell material which formed a coaxial heterojunction with the Au nanowire was created by electrodeposition. Electrical characterization of the Au nanowires revealed resistance variations associated with the viraltemplated assembly process. The photoelectrical response of the core-shell nanowires was used to assess the interaction between the two component materials. A correlation was found between the dark current of the $\mathrm{Au} / \mathrm{CdSe}$ core-shell nanowire and the magnitude of the collected photocurrent.

Keywords: core-shell; nanowire; electrodeposition; M13 virus; electrical properties; nanocrystalline materials

\section{Introduction}

Driven by scaling requirements and the pursuit of novel material properties, nanotechnology has advanced rapidly. Given the shortcomings of man-made tools for precise nanoscale assembly, many researchers have looked to biology for inspiration. The natural world uses biomolecules such as peptides and proteins to expertly direct the assembly of inorganic materials. The organic-inorganic interface controls assembly on multiple length scales ranging from nanoscale to macroscale depending on the required function. Researchers have begun to harness this extraordinary assembly capability to make a variety of devices by integrating peptides or proteins able to bind technologically significant materials into the structural proteins of 
viruses. This approach has allowed the realization of unique device geometries, as well as the opportunity for enhanced performance and functionality.[1-5] One area of viral-assisted assembly that has yet to be fully explored is the formation of core-shell materials. Although ensembles or arrays of viral-templated core-shell nanowires have been studied for battery electrodes or photocurrent collection, discrete viral-templated coreshell nanowires have not yet been reported.[1, 6] Single nanowire studies offer greater insight into the construction of these increasingly complex and hierarchical structures. Here, we investigate individual $\mathrm{Au} / \mathrm{CdSe}$ core-shell nanowires assembled using a filamentous viral scaffold. The high aspect ratio virus served as the template for the seeded electroless deposition of the Au core followed by the electrodeposition of the CdSe shell. The core and shell provided complementary properties: the conductive pathway of a metal nanowire and the efficient photon absorption and carrier generation associated with nanocrystalline semiconductor material. The combination of lithographically patterned substrates, viral-assisted assembly and electrochemical deposition allowed us to provide detailed electrical and photoelectrical analysis of the Au nanowire cores, as well as the resulting Au/CdSe core-shell nanowires. This in turn allowed a better understanding of the interactions between the core and shell materials, in addition to the structural and electrical variability.

\section{Experimental details}

\subsection{Viral-templated Au nanowire formation}

The M13 bacteriophage was used as the template for Au nanowire synthesis. The M13 is a filamentous bacteriophage approximately $890 \mathrm{~nm}$ in length and $6.5 \mathrm{~nm}$ in diameter (supplementary content, Figure S1).[7] Each gold nanowire was formed from a single viral template. Viral-templated Au nanowires were assembled using a procedure which was similar to one previously described.[6] Briefly, silicon substrates with a thermal oxide layer for electrical isolation were pre-patterned with $\mathrm{Ti} / \mathrm{Au}(10 \mathrm{~nm} / 200 \mathrm{~nm})$ contacts which were approximately $500 \mathrm{~nm}$ apart. The substrates were solvent cleaned and activated with oxygen plasma. M13 bacteriophages, genetically engineered to display an 8-mer Au-binding peptide (VSGSSPDS), were deposited onto the substrate immediately after activation by incubating the substrate in a $1.1 \times 10^{6} \mathrm{pfu} / \mu \mathrm{L}$ solution of Au-binding phage.[8] The substrates were washed in 0.7\% Tween, a nonionic detergent, in tris-buffered saline and rinsed in deionized water. The substrates were placed directly in a $5 \mathrm{~nm}$ Au colloid solution at a 
concentration of $5 \times 10^{13}$ particles/mL (Ted Pella) for 1 hour. The Au nanoparticles, which selectively bound to the bacteriophage, were used as seed crystals for the electroless deposition of Au which increased the size, connectivity, and electrical conductivity of the bio-templated Au nanowire wires. Electroless deposition occurred for 3.5 minutes in Nanoprobes GoldEnhance LM solutions A through D.

\subsection{CdSe electrodeposition}

The substrate was immersed in a Petri dish containing the CdSe electrodeposition solution. A coiled Pt wire circumscribing the substrate acted as the counter electrode. A probe station was used to contact a pair of pre-selected pads. A layer of nanocrystalline CdSe was then electrodeposited onto the Au nanowire and the two contact pads it spanned by applying $-0.8 \mathrm{~V}$ (with respect to the Pt counter electrode) to both contact pads for $90 \mathrm{~s}$ unless otherwise stated. A previously reported solution chemistry was used for CdSe

electrodeposition.[9] Briefly, the solution contained cadmium acetate (35 mM), sodium nitrilotriacetate (NTA, $100 \mathrm{mM}$ ), and sodium selenosulfate (50 mM Se dissolved in $100 \mathrm{mM} \mathrm{Na}_{2} \mathrm{SO}_{3}$ using a stock solution of $0.2 \mathrm{M}$ Se stirred for about $2 \mathrm{~h}$ at approximately $60^{\circ} \mathrm{C}$ in a $0.4 \mathrm{M}$ solution of $\mathrm{Na}_{2} \mathrm{SO}_{3}$ ).

\subsection{Characterization}

A scanning electron microscope (SEM, FEI XL40 Sirion or FEI XL30 Sirion) was used to characterize the size and morphology of the Au nanowires and the electrodeposited CdSe. The mean width and standard deviation of each nanowire was computed by measuring the average edge-to-edge distance along the nanowire (see supplementary content). A minimum of 10 nanowires were analyzed at each electrodeposition time to evaluate wire-to-wire variation. The photoelectrical characterization of the samples was performed using two-terminal measurements and a solar simulator with a $300 \mathrm{~W}$ Xe lamp (Oriel). Current-voltage (I-V) measurements were recorded at room temperature both in the dark and under broadband illumination using a source-measure unit (Keithley 2635 SourceMeter). In addition, broadband photocurrent measurements were recorded as a function of time under a $1 \mathrm{~V}$ applied bias, while opening and closing a software-controlled mechanical shutter. A total power density of approximately $50 \mathrm{~mW} / \mathrm{cm}^{2}$ was measured within the 320-620 nm band of the broad spectrum light source.

\section{Results and discussion}


The SEM images in Figure 1 (a-c) show an individual phage-templated Au nanowire bridging the gap between two electrical contact pads, as well as Au nanowires with increasing CdSe electrodeposition times. (See supplementary content, Figure S2 for a low magnification core-shell nanowire image.) The deposition
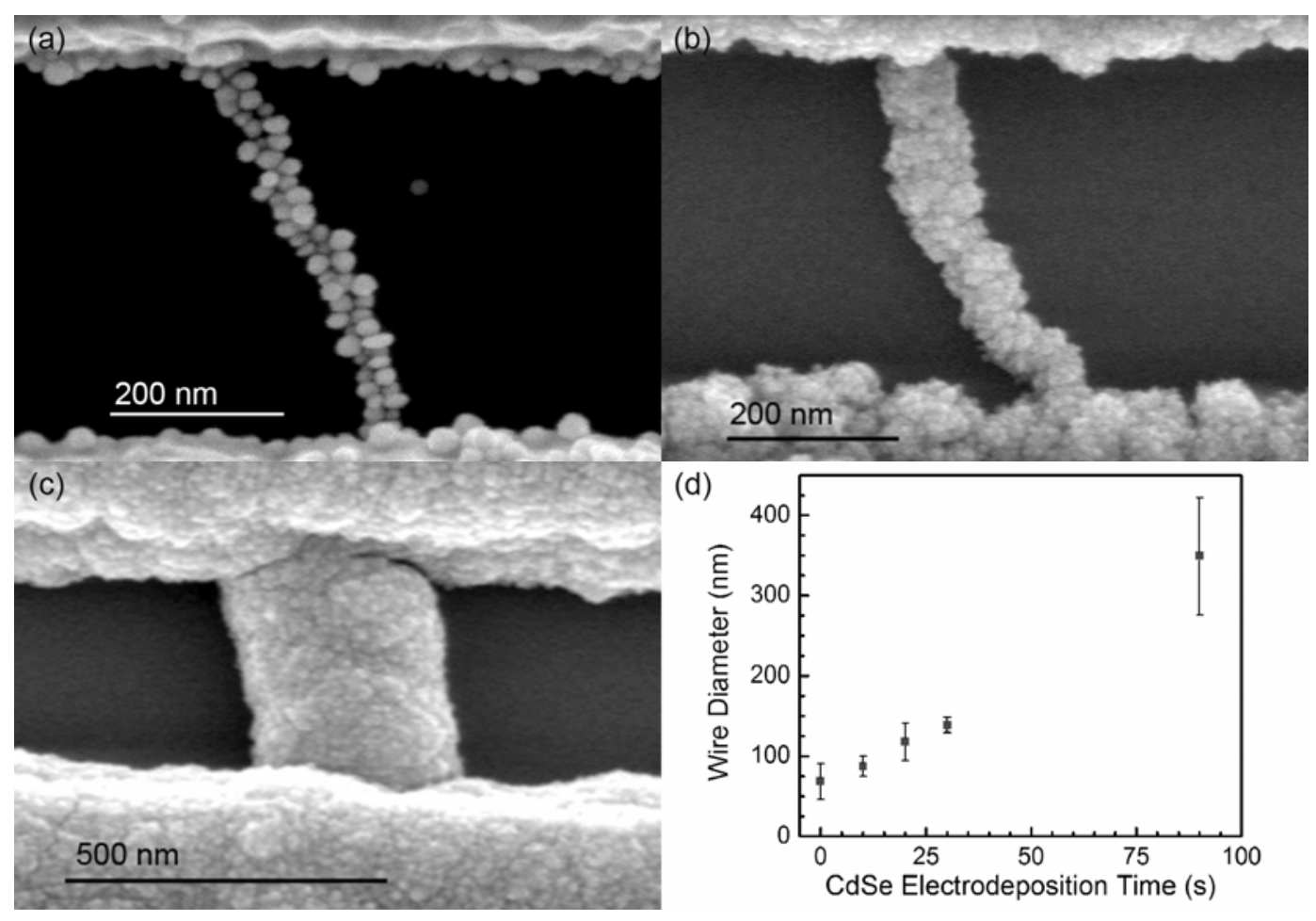

\section{FIGURE 1(a-d)}

time was varied from 0 to $90 \mathrm{~s}$. The templated Au nanowires are polycrystalline with nanoscale grains visible in the SEM and an average width of $64+/-18 \mathrm{~nm}$. This geometry is comparable to other viral-templated $\mathrm{Au}$ nanowires fabricated in a similar manner.[6, 8] The electrodeposited CdSe film is polycrystalline and highly conformal to the underlying Au nanowire geometry. This morphology is consistent with that observed in a previous report using the same selenosulfate-based electrodeposition chemistry and conditions.[9] Furthermore, the deposition was radial to the Au nanowire (supplementary content, Figure S3). The CdSe thickness on the Au nanowire was controlled by the duration of the electrodeposition step. The average measured widths of the nanowires are plotted as a function of electrodeposition time showing wire-to-wire variations in Figure 1(d). The average width variation within an individual core-shell Au/CdSe nanowire is $14 \mathrm{~nm}$ (data not shown) and is relatively independent of electrodeposition time. The CdSe electrodeposition 
partially smoothed the width non-uniformities observed in the Au nanowires, but did not eliminate them completely.
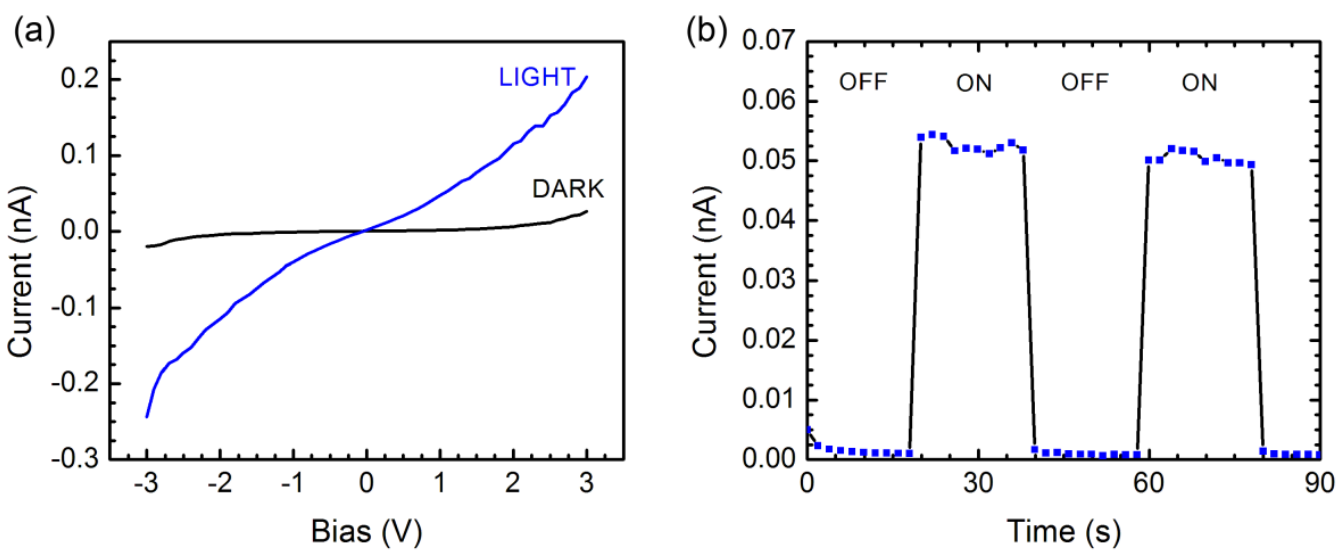

FIGURE 2(a-b)

In order to investigate the resistance variability associated with viral-assembly method, the dark current of almost fifty individual Au nanowire cores was measured with a voltage of $1 \mathrm{~V}$ applied across the two contact pads. Measured currents ranged from open circuit to a few microamps depending on the initial Au nanoparticle coverage of the viral template and the connectivity of the Au nanoparticles within the nanowires following electroless deposition. Many of the Au nanowires exhibited a current decay with a lifetime on the order of 10 s of seconds which was attributed to trap state filling during the measurement. The median current with $1 \mathrm{~V}$ applied bias was $45 \mathrm{nA}$ and currents under $500 \mathrm{nA}$ were found for $\sim 80 \%$ of the measured $\mathrm{Au}$ nanowires. To understand the source of the resistance variations in the viral-templated structures, consider the assembly process. For each nanowire, the initial position and spatial density of the $5 \mathrm{~nm}$ Au seed crystals was slightly different. The phage templates with more tightly packed Au nanoparticles, produced more conductive nanowires than those with larger gaps between Au nanoparticles after the same Au electroless deposition time. Electrical behavior in Au nanowires with low connectivity was likely dominated by electron hopping conduction rather than free electron conduction, resulting in less conductive nanowires. This hypothesis was supported by the non-linear I-V behavior observed for many of the individual Au nanowires as shown in Figure 2(a). In this way, small differences in the initial nanoparticle spacing can lead to large changes in the dark current of the Au nanowire, which later serves as the core of the core-shell nanowire. For 
these experiments, the variations enabled us to readily study the effect of the Au nanowire core resistance on the collection of photogenerated carriers in the CdSe shell.

Photocurrent measurements were made on both the Au nanowire cores alone as well as the Au/CdSe core-shell nanowires. The Au nanowire cores exhibited no electrical response to broad spectrum illumination, whereas a distinct photoresponse was observed in many of the core-shell nanowires. The I-V and

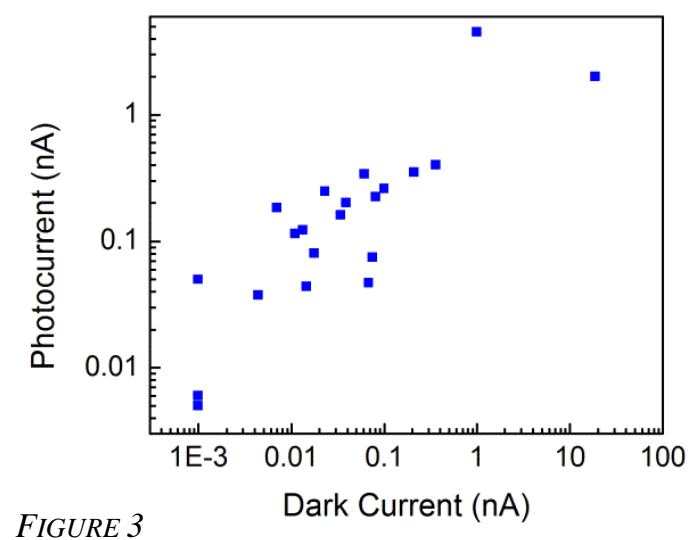

FIGURE 3 photocurrent response of a typical $\mathrm{Au} / \mathrm{CdSe}$ core-shell nanowire is shown in Figure 2(a-b). The slow current decay observed in the photocurrent measurement was attributed to the dark current characteristic of the underlying gold nanowire core. When the core-shell nanowire is illuminated the CdSe absorbs the above bandgap light, generating electron-hole pairs. The photogenerated carriers diffuse to the metal/semiconductor

junction and are collected as photocurrent. The CdSe shell material acts as a photosensitizer while the underlying core material acts a conduit to current flow aiding in the collection of carriers. The electrical interaction between shell and core materials was further investigated by studying the photocurrent as a function of core-shell nanowire dark current, as shown in Figure 3. Collection of photogenerated carriers (or the photocurrent) increased with increasing nanowire conduction. The more conductive Au/CdSe core-shell nanowires were able to more effectively extract and collect the photogenerated carriers as current, reducing recombination losses and increasing the measureable photocurrent. The dependence of photocurrent on dark current is further indication of the electrical interaction between the CdSe shell and the Au nanowire core. If the core and sheath acted as two parallel, independent conduction pathways (as can be modeled by two parallel resistors) the resulting photocurrent would be constant regardless of core nanowire conduction. The dependence of photocurrent on nanowire resistance suggests that either the photogenerated carriers or the energy (i.e. Forster resonant energy transfer) from the photogenerated carriers was transferred from the CdSe shell to the gold nanowire core.[10-12] A measureable photocurrent was observed in about $40 \%$ of the $\mathrm{Au} / \mathrm{CdSe}$ core-shell nanowires. The lack of photocurrent in some nanowires may be caused by disruption of 
connectivity in these fragile nanostructures during electrodeposition or poor adhesion at the $\mathrm{Au} / \mathrm{CdSe}$ interface caused by shrinkage of the CdSe nanocrystalline film during drying.

\section{Conclusion}

In conclusion, individual Au nanowire cores were assembled using a viral-template and electrically characterized. A CdSe shell was then electrodeposited over the Au nanowire core to form single Au/CdSe core-shell nanowires. The CdSe shell material was found to act as a photosensitizer transferring photogenerated carriers or energy to the Au nanowire core for collection. The magnitude of the photocurrent collected depended on the overall resistance of the viral-templated core-shell nanowire.

Acknowledgements: The authors thank the A. M. Belcher lab at MIT for the gift of gold-binding phage and S. Garcia for the M13 drawing. This work was supported by DARPA (Award No. HR0011-07-9-0005).

These studies used the UCSB SSLEC facilities, the MRL Central Facilities supported by the MRSEC Program of the NSF (Award No. DMR05-20415), and the UCSB nanofabrication facility which is part of the NNIN funded by the NSF.

\section{References}

[1] Chen X, Gerasopoulos K, Guo J, Brown A, Wang C, Ghodssi R, et al. Virus-Enabled Silicon Anode for Lithium-Ion Batteries. ACS Nano. 2010;4:5366-72.

[2] Lee YJ, Yi H, Kim W-J, Kang K, Yun DS, Strano MS, et al. Fabricating Genetically Engineered HighPower Lithium-Ion Batteries Using Multiple Virus Genes. Science. 2009;324:1051-5.

[3] Miller RA, Presley AD, Francis MB. Self-Assembling Light-Harvesting Systems from Synthetically Modified Tobacco Mosaic Virus Coat Proteins. Journal of the American Chemical Society. 2007;129:3104-9. [4] Lee BY, Zhang J, Zueger C, Chung W-J, Yoo SY, Wang E, et al. Virus-based piezoelectric energy generation. Nat Nano. 2012;7:351-6.

[5] Atanasova P, Rothenstein D, Schneider JJ, Hoffmann RC, Dilfer S, Eiben S, et al. Virus-Templated Synthesis of ZnO Nanostructures and Formation of Field-Effect Transistors. Advanced Materials. 2011;23:4918-22.

[6] Haberer ED, Joo JH, Hodelin JF, Hu EL. Enhanced photogenerated carrier collection in hybrid films of bio-templated gold nanowires and nanocrystalline CdSe. Nanotechnology. 2009;20:415206. 
[7] Barbas CF, Burton DR, Scott JK, Silverman GJ. Phage Display: A Laboratory Manual. Cold Spring Harbor, New York: Cold Spring Harbor Laboratory Press; 2001.

[8] Huang Y, Chiang C-Y, Lee SK, Gao Y, Hu EL, Yoreo JD, et al. Programmable Assembly of Nanoarchitectures Using Genetically Engineered Viruses. Nano Letters. 2005;5:1429-34.

[9] Hodes G, Grunbaum E, Feldman Y, Bastide S, Levy-Clement C. Variable Optical Properties and Effective Porosity of CdSe Nanocrystalline Films Electrodeposited from Selenosulfate Solutions. Journal of The Electrochemical Society. 2005;152:G917-G23.

[10] Lu S, Lingley Z, Asano T, Harris D, Barwicz T, Guha S, et al. Photocurrent Induced by Nonradiative Energy Transfer from Nanocrystal Quantum Dots to Adjacent Silicon Nanowire Conducting Channels: Toward a New Solar Cell Paradigm. Nano Letters. 2009;9:4548-52.

[11] Shafran E, Mangum BD, Gerton JM. Energy Transfer from an Individual Quantum Dot to a Carbon Nanotube. Nano Letters. 2010;10:4049-54.

[12] Yang T-T, Chen W-T, Hsu Y-J, Wei K-H, Lin T-Y, Lin T-W. Interfacial Charge Carrier Dynamics in Core-Shell Au-CdS Nanocrystals. The Journal of Physical Chemistry C. 2010;114:11414-20.

\section{Figure Captions:}

Figure 1. SEM images showing a viral-templated Au nanowire after increasing CdSe electrodeposition times: (a) no electrodeposition, (b) $20 \mathrm{~s}$, and (c) $90 \mathrm{~s}$. At longer electrodeposition times small cracks were visible in some of the nanowires, as shown in (c) near the junction of the nanowire and contact pad. The average width of the coated nanowires as a function of CdSe electrodeposition time is shown in (d). Error bars represent wire-to-wire variation.

Figure 2. (a) I-V characteristic for a gold/CdSe core/shell nanowire with and without broadband illumination. (b) The broadband photoresponse of an Au/CdSe core-shell nanowire measured as a function of time. The illumination is cycled OFF and ON allowing both the dark current and photocurrent (change in current when illuminated) to be measured with a $1 \mathrm{~V}$ applied bias.

Figure 3. The photocurrent (change in current when illuminated) of several core-shell nanowires under broadspectrum illumination is plotted versus dark current. Both photocurrent and dark current were measured with a $1 \mathrm{~V}$ applied bias. 


\section{Supplementary Content}

Viral-assisted assembly and photoelectric response of individual Au/CdSe core-
shell nanowires

John H. Joo ${ }^{\mathrm{a}}$, Juan F. Hodelin ${ }^{\mathrm{b}}$, Evelyn L. Hu ${ }^{\mathrm{a}}$, and Elaine D. Haberer ${ }^{\mathrm{c}}$

${ }^{a}$ School of Engineering and Applied Sciences, Harvard University, Cambridge, MA 02138 (USA); ${ }^{b}$ Physical Optics Corporation, Torrance, CA 90501 (USA); ${ }^{\mathrm{E}}$ Electrical Engineering Department, Materials Science and Engineering Program, University of California, Riverside, CA 92521 (USA)

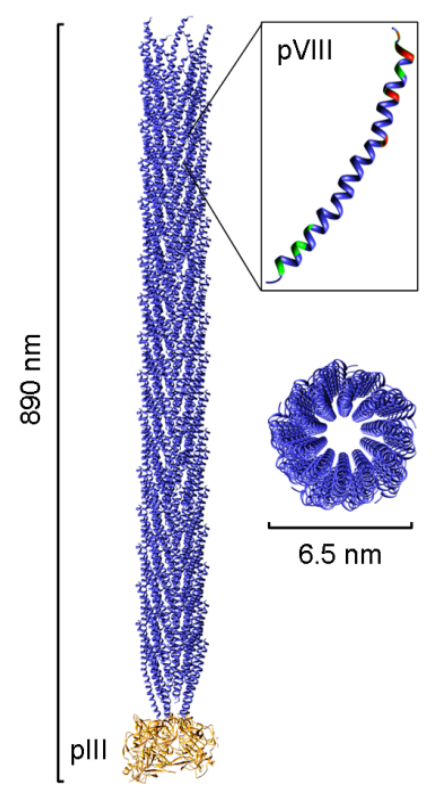

Figure S1. A Chimera drawing (not to scale) of the M13 bacteriophage with the dimensions and geometry of the viral template is shown[S1]. The wild-type virus, approximately $890 \mathrm{~nm}$ long and $6.5 \mathrm{~nm}$ in diameter, is composed of five structural proteins: pIII, pV, pVIII, pIX, and pX. The two proteins most frequently used for phage display applications are shown here. Three to five copies of the pIII (or minor coat protein) are located at the proximal tip of the virus and 2700 copies of the pVIII (or major coat protein) run the length of the virus. (Not all 2700 copies are shown in the drawing.) The genetically modified phages used in these studies have a goldbinding peptide fused to the exposed end of every pVIII protein. 
Nanowire Width Measurement Procedure: The nanowires formed by this viral-templating method displayed large variations in width, therefore the mean width of each wire was determined and used for analysis. To determine the mean width of each nanowire, high magnification SEM images were taken of individual nanowires. Each SEM micrograph was digitally rotated to align along the vertical axis of the image. A Canny edge detector algorithm, a computational algorithm used frequently to define the edges in a wide range of images, was applied to quantitatively define the edges of the nanowire in each image [S2]. Along each pixel down the nanowire, the edge-to-edge distance was measured. The average edgeto-edge distance was reported as the nanowire width.

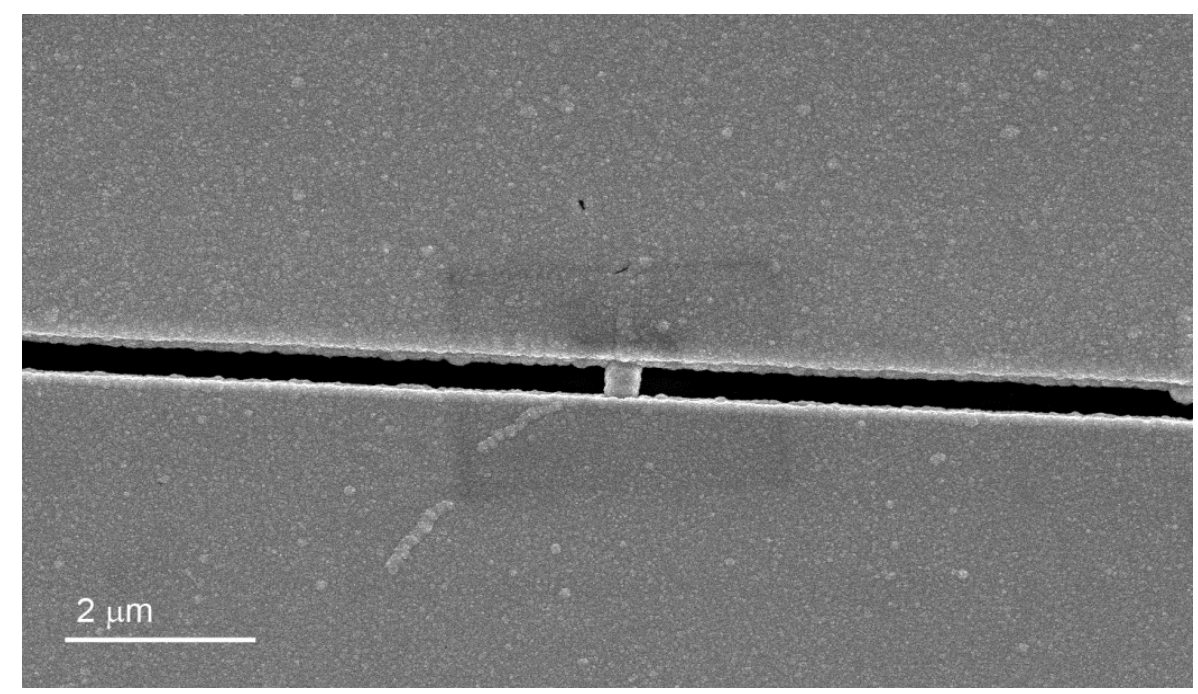

Figure S2. A low magnification SEM image of an Au/CdSe core-shell nanowire.

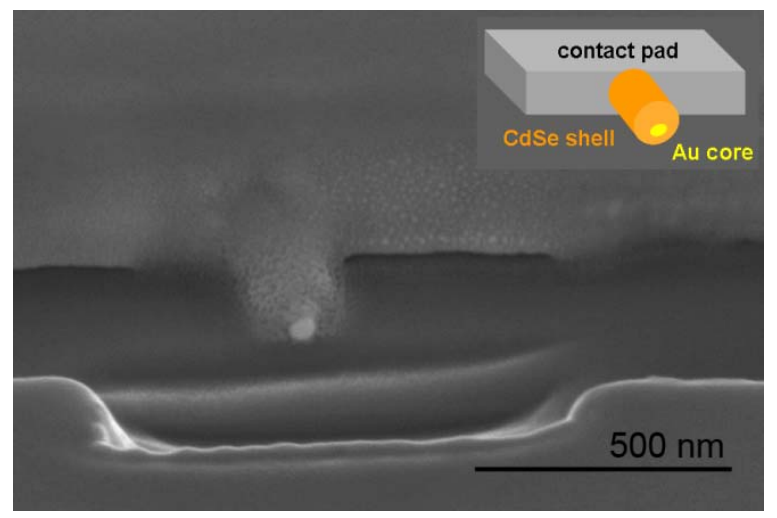

Figure S3. A cross-sectional SEM image of a CdSe coated Au nanowire prepared by FIB showing radial deposition of CdSe. (inset) A schematic of the core-shell nanowire cross-section with the core, shell, and contact pad labeled is shown. 


\section{Acknowledgements}

Molecular graphics and analyses were performed with the UCSF Chimera package. Chimera is developed by the Resource for Biocomputing, Visualization, and Informatics at the University of California, San Francisco, with support from the National Institutes of Health (National Center for Research Resources grant 2P41RR001081, National Institute of General Medical Sciences grant 9P41GM103311).

\section{References}

[S1] Pettersen EF, Goddard TD, Huang CC, Couch GS, Greenblatt DM, Meng EC, et al. UCSF Chimera--a visualization system for exploratory research and analysis. J Comput Chem. 2004;25:1605-12.

[S2] Canny J. A Computational Approach to Edge Detection. Pattern Analysis and Machine Intelligence, IEEE Transactions on. 1986;PAMI-8:679-98. 